\title{
On the Wiener criterion in higher dimensions
}

\section{Tariq Ismaee ${ }^{*}$}

"Correspondence:

tariqismaeel@gcu.edu.pk

School of Mathematics and

Statistics, Xi'an Jiaotong University,

No. 28, Xianning West Road, Xi'an, 710049, China

Department of Mathematics, GC University, Katchery Road, Lahore, 54000, Pakistan

\begin{abstract}
The Wiener criterion is a sufficient and necessary condition for the solvability of the Dirichlet problem. However, its geometric interpretation is not clear. In the case that the domain satisfies an exterior spine condition, the requirement for the spine is clear in dimension 3. In this note, we intend to obtain the condition that the exterior spine should satisfy in higher dimensions.
\end{abstract}

MSC: Primary 35A01; 35B65; 35J05; 35J25

Keywords: Wiener criterion; Laplace equation; Dirichlet problem; boundary regularity

\section{Introduction}

The Laplace equation arises widely in physics and engineering and is a classical prototype of partial differential equations. Consider the following Dirichlet problem for the Laplace equation:

$$
\begin{cases}\Delta u=0 & \text { in } \Omega ; \\ u=g & \text { on } \partial \Omega\end{cases}
$$

where $\Omega \subset R^{n}$ is a bounded domain and $g$ is a continuous function on $\partial \Omega$.

In 1851, Riemann [1] proposed the famous Dirichlet principle, which states that there always exists a harmonic function continuous up to the boundary and coinciding with $g$ on the boundary. However, Lebesgue [2] constructed a bounded domain on which the Dirichlet problem is not always solvable in 1912. By Perron's method [3, 4], there always exists a harmonic function $u$ in $\Omega$ with respect to $g$. If $x_{0} \in \partial \Omega$ is a regular point (see [4, p.25] for the definition), then $u$ is continuous up to $x_{0}$. Hence, the solvability of (1.1) reduces to the problem whether the boundary points are regular. In 1924, Wiener [5] provided a sufficient and necessary condition for the regularity of $x_{0}$. This is the famous Wiener criterion, which solves the Dirichlet problem completely.

However, the geometric interpretation of Wiener criterion is not clear, and it is not easy to verify whether a domain satisfies the Wiener criterion at some boundary point. One of the interesting cases is that the boundary of the domain near some boundary point is constructed by a spine. Precisely, suppose that for a proper coordinate system, $0 \in \partial \Omega$, and there exist $0<r_{0}<1$ and a continuous nondecreasing function $\varphi: R \rightarrow R$ with $\varphi(0)=0$

(c) The Author(s) 2017. This article is distributed under the terms of the Creative Commons Attribution 4.0 International License (http://creativecommons.org/licenses/by/4.0/), which permits unrestricted use, distribution, and reproduction in any medium, provided you give appropriate credit to the original author(s) and the source, provide a link to the Creative Commons license, and indicate if changes were made. 
such that

$$
B_{r_{0}} \cap \Omega=B_{r_{0}} \cap\left\{x \in R^{n}|| x^{\prime} \mid>\varphi\left(x_{n}\right) \text { if } x_{n}>0\right\},
$$

where $B_{r}$ denotes the open ball in $R^{n}$ with radius $r$ and center $0, x=\left(x_{1}, \ldots, x_{n}\right) \in R^{n}$ and $x^{\prime}=\left(x_{1}, \ldots, x_{n-1}\right)$. If ' $=$ ' is replaced by ' $\subset$ ' in (1.2), we call that $\Omega$ satisfies the exterior spine condition with $\varphi$ at 0 .

It is natural to ask what condition $\varphi$ should satisfy to guarantee that 0 is a regular point. It is well known that if $\varphi(r) \geq C r$ for some positive constant $C$, i.e., the domain satisfies the exterior cone condition, then 0 is a regular point (see [6] and [4, Problem 2.12]). In this note, we will consider the weaker condition and always assume that

$$
\varphi(r) / r \rightarrow 0 \quad \text { as } r \rightarrow 0 \quad \text { and } \quad \varphi(r)<r, \quad \forall 0<r<r_{0} .
$$

In the dimension $n=3$, if $\Omega$ satisfies (1.2), it is well known that 0 is a regular point if and only if $\varphi$ satisfies (see Theorem 5.2 and the following example in [7])

$$
\int_{0}^{r_{0}} \frac{d r}{r|\ln \varphi(r)|}=+\infty
$$

What condition $\varphi$ should satisfy in higher dimensions is not known. Besides, the following boundary regularity results are well known. Suppose that $g$ is identically zero on a portion of the boundary near 0 . If $\varphi(r) \geq \mathrm{Cr}^{1 / 2}$, which implies that $\Omega$ satisfies the exterior sphere condition, then the solution is Lipschitz continuous at 0 . If $\varphi(r) \geq C r$, i.e., $\Omega$ satisfies the exterior cone condition, then the solution is Hölder continuous at 0 . A natural question is whether the condition $\varphi(r) \geq \mathrm{Cr}^{a}$ for some constant $C$ and $a>1$ can guarantee the continuity (even Hölder continuity) of the solution at 0 . The geometric meaning of this condition is that $\Omega$ satisfies the exterior Hölder spine condition. It should be pointed out that for the dimension $n=3$, the continuity of the solution in this case is guaranteed (see (1.4)).

This note is devoted to deriving the sufficient and necessary condition for $\varphi$ in a higher dimension and answer the above question.

First, we recall the Wiener criterion. For any bounded domain $\Omega \subset R^{n}$, its capacity is defined by (see Section 2.9 in [4] and Section 19 of Chapter XI in [7])

$$
\operatorname{cap} \Omega=\inf _{v \in K} \int|D v|^{2},
$$

where

$$
K=\left\{v \in C_{0}^{1}\left(R^{n}\right) \mid v=1 \text { on } \Omega\right\}
$$

and $C_{0}^{1}\left(R^{n}\right)$ denotes the set of functions having continuous derivatives and compact support in $R^{n}$.

Suppose that $0 \in \partial \Omega$ and $0<\lambda<r_{0}$ is a constant. Let

$$
\Omega_{j}=\left\{x \notin \Omega\left|\lambda^{j+1} \leq\right| x \mid \leq \lambda^{j}\right\} \quad \text { and } \quad C_{j}=\operatorname{cap} \Omega_{j} \quad \text { for } j=1,2, \ldots
$$


The Wiener criterion states that 0 is a regular point if and only if the series

$$
\sum_{j=1}^{\infty} \frac{C_{j}}{\lambda^{j(n-2)}}
$$

diverges (see Section 2.9 in [4], Section 19 of Chapter XI in [7] and Theorem 5.2 in [7]).

Our main result is the following theorem.

Theorem 1.1 Suppose that $n \geq 4,0 \in \partial \Omega$ and (1.2) is satisfied. Then 0 is a regular point with respect to (1.1) if and only if $\varphi$ satisfies

$$
\int_{0}^{r_{0}} \frac{\varphi^{n-3}(r) d r}{r^{n-2}}=+\infty
$$

An immediate consequence is the following.

Corollary 1.2 Let $n \geq 4$ and $0 \in \partial \Omega$. Suppose that $\Omega$ satisfies the exterior spine condition with $\varphi$ at 0 and (1.6) holds.

Then 0 is a regular point with respect to (1.1).

Remark 1.3 From (1.6), the special dimensions $n=2,3$ should be noted. In addition, for $n \geq 4$ and $a>1, \varphi(r) \geq \mathrm{Cr}^{a}$ is not enough to guarantee that 0 is a regular point, which is an essential difference to dimensions 2 and 3 .

\section{Proof of Theorem 1.1}

Now, we give the proof of Theorem 1.1.

Proof We use ellipsoids to approximate $\Omega_{j}$ and hence to estimate $C_{j}$. Clearly, $\Omega_{j}$ is contained in an ellipsoid $E_{j}$ with semi-axes $2 \lambda^{j}$ and $2 \varphi\left(\lambda^{j}\right)$ ( $n-1$ repeats), and $\Omega_{j}$ contains an ellipsoid $\tilde{E}_{j}$ with semi-axes $\lambda^{j+1}$ and $\varphi\left(\lambda^{j+1}\right) / 2$ ( $n-1$ repeats). The capacity for this kind ellipsoid $E$ is (see (125) in [8])

$$
\operatorname{cap} E=\left(\beta^{2}-\gamma^{2}\right)^{(n-2) / 2} / \int_{0}^{\arcsin (\sqrt{\mu})} \frac{\sin ^{n-3} \theta}{\cos ^{n-2} \theta} d \theta,
$$

where $\beta$ and $\gamma$ are semi-axes of $E$ with $\beta>\gamma$ and $\mu=1-\gamma^{2} / \beta^{2}$. Next, let

$$
I_{m, k}=\int_{0}^{\arcsin (\sqrt{\mu})} \frac{\sin ^{m} \theta}{\cos ^{k} \theta} d \theta .
$$

Then $I_{m, k}$ has the following reduction formula for $k \neq 1$ (see (155) in [8]):

$$
I_{m, k}=\frac{\mu^{(m-1) / 2}}{(k-1)(1-\mu)^{(k-1) / 2}}-\frac{m-1}{k-1} I_{m-2, k-2} .
$$

We say that $A \simeq B$ if $A_{1} A \leq B \leq A_{2} A$, where $A_{1}$ and $A_{2}$ are constants depending only on the dimension $n$. If $1-\mu \ll 1$, then

$$
\frac{\mu^{(m-1) / 2}}{(k-1)(1-\mu)^{(k-1) / 2}} \simeq(1-\mu)^{-(k-1) / 2} .
$$


Substitute $m=n-3$ and $k=n-2$ and, by noting for $k=1$ (see [9, Section 442.10])

$$
I_{0,1}=\frac{1}{2} \ln \frac{1+\sqrt{\mu}}{1-\sqrt{\mu}}
$$

we have

$$
I_{n-3, n-2} \simeq(1-\mu)^{-(n-3) / 2} .
$$

Therefore, substituting into (2.1) leads to

$$
\operatorname{cap} E \simeq\left(\beta^{2}-\gamma^{2}\right)^{(n-2) / 2} \frac{\gamma^{n-3}}{\beta^{n-3}} \simeq \beta \gamma^{n-3} .
$$

Let $E=E_{j}$ with $\beta=2 \lambda^{j}$ and $\gamma=2 \varphi\left(\lambda^{j}\right)$, then $1-\mu \ll 1$ for sufficient large $j$ (recall (1.3)) and hence

$$
C_{j}=\operatorname{cap} \Omega_{j} \leq \operatorname{cap} E_{j} \leq A_{1} \lambda^{j} \varphi^{n-3}\left(\lambda^{j}\right) .
$$

Similarly, let $E=\tilde{E}_{j}$ with $\beta=\lambda^{j+1}$ and $\gamma=\varphi\left(\lambda^{j+1}\right) / 2$, then

$$
C_{j}=\operatorname{cap} \Omega_{j} \geq \operatorname{cap} \tilde{E}_{j} \geq A_{2} \lambda^{j+1} \varphi^{n-3}\left(\lambda^{j+1}\right) .
$$

Note that

$$
\sum_{j=1}^{\infty} \frac{\lambda^{j} \varphi^{n-3}\left(\lambda^{j}\right)}{\lambda^{j(n-2)}}=\infty
$$

is equivalent to

$$
\sum_{j=1}^{\infty} \frac{\lambda^{j+1} \varphi^{n-3}\left(\lambda^{j+1}\right)}{\lambda^{(j+1)(n-2)}}=\infty
$$

Hence, (1.5) holds if and only if

$$
\sum_{j=1}^{\infty} \frac{\lambda^{j} \varphi^{n-3}\left(\lambda^{j}\right)}{\lambda^{j(n-2)}}=\infty
$$

whose integral representation is exactly (1.6). Therefore, 0 is a regular point if and only if (1.6) holds and hence Theorem 1.1 is completed.

\section{Conclusion}

Let $\Omega$ be a bounded domain in $R^{n}$ and $x_{0} \in \partial \Omega$. The Wiener criterion provides a necessary and sufficient condition on $\Omega$ to solve the Dirichlet problem of the Laplace equation (i.e., (1.1)). However, its geometric meaning is not clear and it is not easy to verify whether a domain satisfies the Wiener criterion at some boundary point. One interesting case is that $\Omega$ is constructed by a spine near $x_{0}$ (see (1.2)). It is natural to ask what condition the spine 
should satisfy to guarantee that $x_{0}$ is a regular point. When the dimension $n=2$ or 3 , the results are well known. In this paper, we consider $n>3$ and prove that $x_{0}$ is a regular point with respect to the Dirichlet problem if and only if the spine satisfies (1.6).

Funding

This research is supported by NSFC 11671316.

Availability of data and materials

Not applicable.

Ethics approval and consent to participate

Not applicable.

\section{Competing interests}

The author declares that they have no competing interests.

\section{Consent for publication}

Not applicable.

\section{Authors' contributions}

All authors read and approved the final manuscript.

\section{Publisher's Note}

Springer Nature remains neutral with regard to jurisdictional claims in published maps and institutional affiliations.

Received: 9 July 2017 Accepted: 13 November 2017 Published online: 21 November 2017

\section{References}

1. Brezis, H, Browder, F: Partial differential equations in the 20th century. Adv. Math. 135(1), 76-144 (1998). doi:10.1006/aima.1997.1713

2. Lebesgue, H: Sur des cas d'impossibilite du probleme de Dirichlet ordinaire. C.R. Seances Soc. Math. France 17 (1912)

3. Perron, O: Eine neue Behandlung der ersten Randwertaufgabe für $\Delta u=0$. Math. Z. 18(1), $42-54$ (1923). doi:10.1007/BF01192395

4. Gilbarg, D, Trudinger, NS: Elliptic Partial Differential Equations of Second Order. Classics in Mathematics. Springer, Berlin (2001)

5. Wiener, N: The Dirichlet problem. J. Math. Phys. 3(3), 127-146 (1924)

6. Miller, K: Barriers on cones for uniformly elliptic operators. Ann. Mat. Pura Appl. (4) 76, 93-105 (1967). doi:10.1007/BF02412230

7. Kellogg, OD: Foundations of Potential Theory. Die Grundlehren der Mathematischen Wissenschaften, vol. 31. Springer, Berlin (1929). Reprint from the first edition of 1929

8. Tee, GJ: Surface area and capacity of ellipsoids in $n$ dimensions. N.Z. J. Math. 34(2), 165-198 (2005)

9. Dwight, HB: Tables of Integrals and Other Mathematical Data, 3rd edn. Macmillan Co., New York (1957)

\section{Submit your manuscript to a SpringerOpen ${ }^{0}$ journal and benefit from:}

- Convenient online submission

- Rigorous peer review

- Open access: articles freely available online

High visibility within the field

- Retaining the copyright to your article 